PROCEEDINGS OF THE

AMERICAN MATHEMATICAL SOCIETY

Volume 132, Number 2, Pages 429-434

S 0002-9939(03)07076-X

Article electronically published on June 17, 2003

\title{
TWO NONTRIVIAL SOLUTIONS FOR QUASILINEAR PERIODIC EQUATIONS
}

\author{
EVGENIA H. PAPAGEORGIOU AND NIKOLAOS S. PAPAGEORGIOU \\ (Communicated by Carmen C. Chicone)
}

\begin{abstract}
In this paper we study a nonlinear periodic problem driven by the ordinary scalar p-Laplacian and with a Carathéodory nonlinearity. We establish the existence of at least two nontrivial solutions. Our approach is variational based on the smooth critical point theory and using the "Second Deformation Theorem".
\end{abstract}

\section{INTRODUCTION}

In the last decade there has been increasing interest for periodic problems driven by the ordinary p-Laplacian differential operator. We refer to the works of Del Pino-Manasevich-Murua [5], Fabry-Fayyad [6], Guo [7, Dang-Oppenheimer [4] (scalar problems), and Manasevich-Mawhin [10, Mawhin [11], Kyritsi-MatzakosPapageorgiou [9] (vector problems). In all these works the method of analysis is based on degree theoretic arguments or on the theory of nonlinear operators of monotone type and on fixed point results (see Kyritsi-Matzakos-Papageorgiou [9]). The problem of existence of multiple periodic solutions was addressed only by Del Pino-Manasevich-Murua [5], where the forcing term $f(t, x)$ is continuous, the map $x \rightarrow \hat{f}(t, x)=f\left(t,\|x\|^{p-2} x\right)$ is locally Lipschitz, and if $p>2, f(t, x) \neq 0$ for all $x \neq 0$. Their approach is based on conditions on the interaction between the Fučik spectrum of the ordinary p-Laplacian and the nonlinearity $f$.

In this paper we prove a multiplicity result for problems with a Carathéodory nonlinearity. We assume that the equation is strongly resonant at the first (zero) eigenvalue of the negative ordinary scalar p-Laplacian with periodic boundary conditions (i.e., $f(t, x) \rightarrow 0$ as $|x| \rightarrow \infty$ and the potential $F(t, x)=\int_{0}^{x} f(t, r) d r$ has finite limits as $|x| \rightarrow \infty$, i.e., the potential has a small rate of increase as $|x| \rightarrow \infty)$. The term "strong resonance" (describing the situation just mentioned) was coined by Bartolo-Benci-Fortunato [2. Our approach is variational and uses smooth critical point theory (see Chang [3] and Mawhin-Willem [12]) and the so-called "second deformation theorem" (see Chang [3], p.23).

Received by the editors May 29, 2002 and, in revised form, September 30, 2002.

2000 Mathematics Subject Classification. Primary 34B15, 34C25.

Key words and phrases. Ordinary p-Laplacian, critical point, Palais-Smale condition, second deformation theorem, strong deformation retract, strong resonance.

(C)2003 American Mathematical Society 


\section{Multiplicity Result}

The problem under consideration is the following:

$$
\left\{\begin{array}{l}
-\left(\left|x^{\prime}(t)\right|^{p-2} x^{\prime}(t)\right)^{\prime}=f(t, x(t)) \text { a.e on } T=[0, b] \\
x(0)=x(b), x^{\prime}(0)=x^{\prime}(b), 1<p<\infty .
\end{array}\right\}
$$

Our hypotheses on the nonlinearity $f$ are the following:

$H(f) . f: T \times \mathbb{R} \longmapsto \mathbb{R}$ is a function such that:

(i) for all $x \in \mathbb{R}, t \longmapsto f(t, x)$ is measurable;

(ii) for almost all $t \in T$, the function $x \longmapsto f(t, x)$ is continuous;

(iii) for almost all $t \in T$ and all $x \in \mathbb{R}$, we have

$$
|f(t, x)| \leq a_{1}(t)+c_{1}(t)|x|^{r-1},
$$

$1 \leq r<+\infty$ with $a_{1}, c_{1} \in L^{r^{\prime}}(T), \frac{1}{r}+\frac{1}{r^{\prime}}=1$;

(iv) if $F(t, x)=\int_{0}^{x} f(t, r) d r$, there exist $F_{ \pm} \in L^{1}(T)$ such that $F(t, x)$ $\rightarrow F_{ \pm}(t)$ as $x \rightarrow \pm \infty$ uniformly for almost all $t \in T, \int_{0}^{b} F_{ \pm}(t) d t \leq 0$;

(v) for almost all $t \in T$ and all $x \in \mathbb{R}, F(t, x) \leq \frac{1}{b^{p} p}|x|^{p}$;

(vi) $\liminf _{x \rightarrow 0} \frac{p F(t, x)}{|x|^{p}} \geq \theta(t)$ uniformly for almost all $t \in T$ with $\theta \in L^{1}(T)$ and $\int_{0}^{b} \theta(t) d t>0$.

We introduce the space $W_{p e r}^{1, p}(T)=\left\{x \in W^{1, p}(T): x(0)=x(b)\right\}$ and the functional $\varphi: W_{p e r}^{1, p}(T) \rightarrow \mathbb{R}$ defined by $\varphi(x)=\frac{1}{p}\left\|x^{\prime}\right\|_{p}^{p}-\int_{0}^{b} F(t, x(t)) d t$. It is well known that $\varphi \in C^{1}\left(W_{p e r}^{1, p}(T)\right)$. Recall that if $X$ is a Banach space and $\varphi \in C^{1}(X)$, we say that $\varphi$ satisfies the Palais-Smale condition at level $c\left((\mathrm{PS})_{c}\right.$-condition for short) if every sequence $\left\{x_{n}\right\}_{n \geq 1}$ such that $\varphi\left(x_{n}\right) \rightarrow c$ and $\varphi^{\prime}\left(x_{n}\right) \rightarrow 0$ has a strongly convergent subsequence. In what follows for $\varphi \in C^{1}(X)$, we set $K=\left\{x \in X: \varphi^{\prime}(x)=0\right\}$ (the set of critical points of $\varphi$ ) and for $c \in \mathbb{R}, K_{c}=\left\{x \in X: \varphi^{\prime}(x)=0\right.$ and $\varphi(x)=c\}$ and $\varphi^{c}=\{x \in X: \varphi(x) \leq c\}$. The so-called "second deformation theorem" (see Chang [3], p. 23) that we shall use says the following:

Theorem 1. If $X$ is a Banach space, $\varphi \in C^{1}(X)$ satisfies the $(P S)_{c}$-condition for every $c \in[a, d]$, $a$ is the only critical value of $\varphi$ on $[a, d)$ and $\varphi^{-1}(\{a\}) \cap K$ consists of isolated critical points, then there exists $h \in C\left([0,1] \times \varphi^{d} \backslash K_{d}, X\right)$ such that $\left.h(t, \cdot)\right|_{\varphi^{a}}=$ identity for all $t \in T, h(0, \cdot)=$ identity and $h\left(1, \varphi^{d} \backslash K_{d}\right) \subseteq \varphi^{a}$. In addition $\varphi(h(s, x)) \leq \varphi(h(t, x))$ for all $t<s$ and all $x \in \varphi^{d} \backslash K_{d}$.

Remark. In the terminology of Chang [3] and Mawhin-Willem [12] (p. 171), the first part of the conclusion of Theorem 1 says that $\varphi^{a}$ is a strong deformation retract of $\varphi^{d} \backslash K_{d}$

Proposition 2. If hypotheses $H(f)$ hold, then for every $c<-\int_{0}^{b} F_{ \pm}(t) d t$, $\varphi$ satisfies the $(P S)_{c}$-condition.

Proof. Let $\left\{x_{n}\right\}_{n \geq 1} \subseteq W_{\text {per }}^{1, p}(T)$ be a sequence such that $\varphi\left(x_{n}\right) \rightarrow c$ and $\varphi^{\prime}\left(x_{n}\right) \rightarrow 0$. We have $\varphi^{\prime}\left(x_{n}\right)=A\left(x_{n}\right)-N\left(x_{n}\right)$ where $A: W_{p e r}^{1, p}(T) \rightarrow W_{p e r}^{1, p}(T)^{*}$ is the nonlinear operator defined by $\langle A(x), y\rangle=\int_{0}^{b}\left|x^{\prime}(t)\right|^{p-2} x^{\prime}(t) y^{\prime}(t) d t$ for all $x, y \in W_{\text {per }}^{1, p}(T)$ (by $\langle\cdot, \cdot\rangle$ we denote the duality brackets for the pair $\left(W_{p e r}^{1, p}(T), W_{p e r}^{1, p}(T)^{*}\right)$ and $N$ : $L^{r}(T) \rightarrow L^{r^{\prime}}(T)$ is the Nemitsky operator corresponding to $f$, i.e., $N(x)(\cdot)=$ $f(\cdot, x(\cdot))$. It is easy to check that $A$ is monotone, demicontinuous, hence maximal 
monotone (see Hu-Papageorgiou [8], p. 309) and $N$ is continuous (Krasnoselkii's theorem).

We claim that the sequence $\left\{x_{n}\right\}_{n \geq 1} \subseteq W_{\text {per }}^{1, p}(T)$ is bounded. Suppose that this is not the case. By passing to a subsequence if necessary, we may assume that $\left\|x_{n}\right\| \rightarrow \infty$. Set $y_{n}=\frac{x_{n}}{\left\|x_{n}\right\|}, n \geq 1$. We may assume that $y_{n} \stackrel{w}{\rightarrow} y$ in $W_{p e r}^{1, p}(T)$ and $y_{n} \rightarrow y$ in $C(T)$ (recall that by the Sobolev embedding theorem $W_{p e r}^{1, p}(T)$ is embedded compactly in $C(T)$ ). From the choice of the sequence $\left\{x_{n}\right\}_{n \geq 1} \subseteq$ $W_{p e r}^{1, p}(T)$, we have

$$
\frac{\left|\varphi\left(x_{n}\right)\right|}{\left\|x_{n}\right\|^{p}}=\left|\frac{1}{p}\left\|y_{n}^{\prime}\right\|_{p}^{p}-\int_{0}^{b} \frac{F\left(t, x_{n}(t)\right)}{\left\|x_{n}\right\|^{p}}\right| d t \leq \frac{M_{1}}{\left\|x_{n}\right\|^{p}}
$$

for some $M_{1}>0$ and all $n \geq 1$.

Because of hypothesis $H(f)\left(\right.$ iv), we can find $M_{2}>0$ such that for almost all $t \in T$, we have that

$$
|F(t, x)| \leq\left|F_{+}(t)\right|+1 \text { if } x \geq M_{2} \text { and }|F(t, x)| \leq\left|F_{-}(t)\right|+1 \text { if } x \leq-M_{2} .
$$

From the mean value theorem we know that for almost all $t \in T$ and all $x \in \mathbb{R}$ we can find $\lambda=\lambda(t, x) \in(0,1)$ such that $F(t, x)=f(t, \lambda x) x$. Then using hypothesis $H(f)$ (iii), we see that for almost all $t \in T$ and all $x \in\left(-M_{2}, M_{2}\right)$ we have $|F(t, x)| \leq$ $a_{2}(t)$ with $a_{2} \in L^{r^{\prime}}(T)$. So finally for almost all $t \in T$ and all $x \in \mathbb{R}$, we have $|F(t, x)| \leq a_{3}(t)$ with $a_{3} \in L^{1}(T)$. Therefore $\left|\int_{0}^{b} \frac{F\left(t, x_{n}(t)\right)}{\left\|x_{n}\right\|^{p}} d t\right| \leq \int_{0}^{b} \frac{a_{3}(t)}{\left\|x_{n}\right\|^{p}} d t \rightarrow 0$ as $n \rightarrow \infty$. Hence if we pass to the limit in (2), we obtain $\frac{1}{p}\left\|y^{\prime}\right\|_{p}^{p}=0$ and so $y=\xi \in \mathbb{R}$. If $\xi=0$, then $y_{n}^{\prime} \rightarrow 0$ in $L^{p}(T)$ and so $y_{n} \rightarrow 0$ in $W_{p e r}^{1, p}(T)$, a contradiction since $\left\|y_{n}\right\|=1$ for all $n \geq 1$. So $\xi \neq 0$ and without any loss of generality we may assume that $\xi>0$ (the analysis is similar if instead we assume that $\xi<0)$. From the choice of the sequence $\left\{x_{n}\right\}_{n \geq 1} \subseteq W_{p e r}^{1, p}(T)$, we know that there exists $n_{0} \geq 1$ such that for all $n \geq n_{0}$, we have $\left|\varphi\left(x_{n}\right)-c\right| \leq \varepsilon$, hence $\varphi\left(x_{n}\right) \leq c+\varepsilon$ and so $-\int_{0}^{b} F\left(t, x_{n}(t)\right) d t \leq c+\varepsilon$. Note that $x_{n}(t) \rightarrow+\infty$ uniformly in $t \in T$ and so $F\left(t, x_{n}(t)\right) \rightarrow F_{+}(t)$ uniformly for almost all $t \in T$ (hypothesis $H(f)(\mathrm{iv}))$. Therefore $-\int_{0}^{b} F_{+}(t) d t \leq c+\varepsilon$. Let $\varepsilon \downarrow 0$ to obtain $-\int_{0}^{b} F_{+}(t) d t \leq c$, a contradiction to the choice of $c$. Similarly we reach a contradiction if we assume that $\xi<0$.

Therefore we have proved that $\left\{x_{n}\right\}_{n \geq 1} \subseteq W_{\text {per }}^{1, p}(T)$ is bounded and so we may assume that $x_{n} \stackrel{w}{\longrightarrow} x$ in $W_{p e r}^{1, p}(T)$ and $x_{n} \rightarrow x$ in $C(T)$. From the choice of the sequence $\left\{x_{n}\right\}_{n \geq 1} \subseteq W_{\text {per }}^{1, p}(T)$ we have

$$
\left\langle A\left(x_{n}\right), x_{n}-x\right\rangle-\int_{0}^{b} f\left(t, x_{n}(t)\right)\left(x_{n}(t)-x(t)\right) d t \leq \varepsilon_{n}\left\|x_{n}-x\right\| \text { with } \varepsilon_{n} \downarrow 0 .
$$

Note that by virtue of hypothesis $H(f)$ (iii) and the dominated convergence theorem, we have that $\int_{0}^{b} f\left(t, x_{n}(t)\right)\left(x_{n}(t)-x(t)\right) d t \rightarrow 0$. So from (3) we obtain that $\lim \sup \left\langle A\left(x_{n}\right), x_{n}-x\right\rangle \leq 0$. But $A$ being maximal monotone, it is generalized pseudomonotone (see Hu-Papageorgiou [8], p. 365) and so $\left\langle A\left(x_{n}\right), x_{n}\right\rangle \rightarrow\langle A(x), x\rangle$, hence $\left\|x_{n}^{\prime}\right\|_{p} \rightarrow\left\|x^{\prime}\right\|_{p}$. Also $x_{n}^{\prime} \stackrel{w}{\longrightarrow} x^{\prime}$ in $L^{p}(T)$ and since the latter is uniformly convex, by the Kadec-Klee property we have $x_{n}^{\prime} \rightarrow x^{\prime}$ in $L^{p}(T)$. Therefore $x_{n} \rightarrow x$ in $W_{p e r}^{1, p}(T)$.

Now we are ready for the multiplicity result. 
Theorem 3. If hypotheses $H(f)$ hold, then there exist two nontrivial solutions $x_{1}, x_{2} \in C^{1}(T)$ of (1) such that $\left|x_{i}^{\prime}(\cdot)\right|^{p-2} x_{i}^{\prime}(\cdot) \in W_{\text {per }}^{1, \tau}(T)$ with $\tau=\min \left\{q, r^{\prime}\right\}$ and $i=1,2$.

Proof. Note that because of hypotheses $H(f)$ (iii) and (iv), $\varphi$ is bounded below. Also hypothesis $H(f)$ (vi) implies that given $\varepsilon>0$, we can find $\delta(\varepsilon)>0$ such that for almost all $t \in T$ and all $|x| \leq \delta$, we have

$$
(\theta(t)-\varepsilon)|x|^{p} \leq p F(t, x) .
$$

For every $\xi \in \mathbb{R}$, we have

$$
\varphi(\xi)=-\int_{0}^{b} F(t, \xi) d t \leq \frac{|\xi|^{p}}{p} \int_{0}^{b}(\varepsilon-\theta(t)) d t=\frac{|\xi|^{p} \varepsilon b}{p}-\frac{|\xi|^{p}}{p} \int_{0}^{b} \theta(t) d t .
$$

From the properties of $\theta$ (see hypothesis $H(f)(\mathrm{vi})$ ), we see that if $\varepsilon>0$ is small, $\varphi(\xi)<0$. So we infer that $\inf \left[\varphi(x): x \in W_{\text {per }}^{1, p}(T)\right]=m_{0}<0 \leq-\int_{0}^{b} F_{ \pm}(t) d t$ (see hypothesis $H(f)(\mathrm{iv})$ ). According to Proposition 2, $\varphi$ satisfies the (PS) $m_{m_{0}}$-condition. Thus we can find $x_{1} \in W_{p e r}^{1, p}(T)$ such that

$$
\varphi\left(x_{1}\right)=m_{0}<0=\varphi(0) \text {, hence } \varphi^{\prime}\left(x_{1}\right)=0 \text { and } x_{1} \neq 0 .
$$

Suppose that $x_{1}$ and 0 are the only critical points of $\varphi$. From the above argument we know that given $\varepsilon>0$, we can find $r_{1}>0$ such that if $|\xi| \leq r_{1}$, we have

$$
\varphi(\xi) \leq \frac{|\xi|^{p}}{p}\left(\varepsilon b-\int_{0}^{b} \theta(t) d t\right) .
$$

If $\varepsilon>0$ is small, we have that $\eta=\varepsilon b-\int_{0}^{b} \theta(t) d t<0$ and so

$$
\varphi(\xi) \leq \frac{|\xi|^{p}}{p} \eta<0 .
$$

Consider the direct sum decomposition $W_{\text {per }}^{1, p}(T)=\mathbb{R} \oplus V$ with $V=\{v \in$ $\left.W_{p e r}^{1, p}(T): \int_{0}^{b} v(t) d t=0\right\}$. By virtue of hypothesis $H(f)(\mathrm{v})$, for every $v \in V$, we have

$$
\varphi(v) \geq \frac{1}{p}\left\|v^{\prime}\right\|_{p}^{p}-\frac{1}{p b^{p}}\|v\|_{p}^{p}
$$

From the Wirtinger inequality (see Mawhin-Willem [12], p. 8), we have $\|v\|_{p}^{p} \leq$ $b\|v\|_{\infty}^{p} \leq b^{p}\left\|v^{\prime}\right\|_{p}^{p}$ and so

$$
\varphi(v) \geq \frac{1}{p}\left\|v^{\prime}\right\|_{p}^{p}-\frac{1}{p}\left\|v^{\prime}\right\|_{p}^{p}=0, \text { i.e., } \quad \inf _{V} \varphi=0 .
$$

From the previous considerations (see (5)), we know that

$$
\mu=\sup _{\bar{B}_{r} \cap \mathbb{R}} \varphi<0 .
$$

Here $B_{r}=\left\{x \in W_{\text {per }}^{1, p}(T):\|x\|<r\right\}$. Let $\Gamma=\left\{\gamma \in C\left(\bar{B}_{r} \cap \mathbb{R}, W_{\text {per }}^{1, p}(T)\right)\right.$ : $\left.\gamma\right|_{\partial \bar{B}_{r} \cap \mathbb{R}}=$ identity $\}$. If $h$ is the homotopy postulated by Theorem 1 , we define the map $\gamma_{0}: \bar{B}_{r} \cap \mathbb{R} \rightarrow W_{\text {per }}^{1, p}(T)$ by

$$
\gamma_{0}(x)= \begin{cases}x_{1} & \text { if }\|x\|<\frac{r}{2}, \\ h\left(\frac{2(r-|x|)}{r}, \frac{r x}{\|x\|}\right) & \text { if }\|x\| \geq \frac{r}{2} .\end{cases}
$$

Recall that we have assumed that $\left\{x_{1}, 0\right\}$ are the only critical points of $\varphi$. Then $x_{1}$ is the only minimizer of $\varphi$ and so from Theorem 1 , it follows that $h(1, y)=x_{1}$ 
for all $y \in \varphi^{0} \backslash\{0\}$. Hence we infer that for $|x|=\frac{r}{2}$, we have $h\left(\frac{2(r-|x|)}{r}, \frac{r x}{\|x\|}\right)=$ $h(1,2 x)=x_{1}$, which proves the continuity of $\gamma_{0}$. In addition, from Theorem 1 , $h(0, \cdot)=$ identity and so $\left.\gamma_{0}\right|_{\partial \bar{B}_{r} \cap \mathbb{R}}=$ identity. Thus it follows that $\gamma_{0} \in \Gamma$. Moreover, since $h$ is $\varphi$-decreasing (see Theorem 1), for all $x \in \varphi^{0} \backslash\{0\}$ and all $t, s \in[0,1]$ with $t<s$, we have $\varphi(h(s, x)) \leq \varphi(h(t, x))$. From this and $(6)$, we see that

$$
\varphi\left(\gamma_{0}(x)\right)<0 \text { for all } x \in \bar{B}_{r} \cap \mathbb{R} \text {. }
$$

From Struwe [13] (p. 116), we know that the sets $\partial \bar{B}_{r} \cap \mathbb{R}$ and $V$ link. So we have that $\gamma\left(\bar{B}_{r} \cap \mathbb{R}\right) \cap V \neq 0$ for all $\gamma \in \Gamma$, hence $\sup \left[\varphi(\gamma(x)): x \in \bar{B}_{r} \cap \mathbb{R}\right] \geq 0$ for all $\gamma \in \Gamma$ (recall that $\inf _{V} \varphi=0$ ) and so

$$
\sup \left[\varphi\left(\gamma_{0}(x)\right): x \in \bar{B}_{r} \cap \mathbb{R}\right]=\varphi\left(\gamma_{0}\left(x^{*}\right)\right) \geq 0 \text { for some } x^{*} \in \bar{B}_{r} \cap \mathbb{R} .
$$

From (7) and (8), we have a contradiction. Therefore $\varphi$ has another critical point $x_{2} \neq x_{1}, x_{2} \neq 0$. Now let $y=x_{k}, k=1,2$. We have $\varphi^{\prime}(y)=0$ and so

$$
A(y)=N(y)
$$

hence $\langle A(y), \psi\rangle=\int_{0}^{b} f(t, y(t)) \psi(t) d t$ for all $\psi \in C_{0}^{\infty}(0, b)$.

Note that $\left|y^{\prime}(\cdot)\right|^{p-2} y^{\prime}(\cdot) \in W^{-1, q}(T)=W_{0}^{1, p}(T)^{*}$ (see Adams [1], p. 50). So from the definition of distributional derivative we have $\left\langle-\left(\left|y^{\prime}\right|^{p-2} y^{\prime}\right)^{\prime}, \psi\right\rangle_{0}=\langle N(y), \psi\rangle_{0}$, with $\langle\cdot, \cdot\rangle_{0}$ denoting the duality brackets for the pair $\left.\left(W_{0}^{1, p}\right)(T), W^{-1, q}(T)\right)$. Since $C_{0}^{\infty}(0, b)$ is dense in $W_{0}^{1, p}(T)$, we have

$$
-\left(\left|y^{\prime}(t)\right|^{p-2} y^{\prime}(t)\right)^{\prime}=f(t, y(t)) \text { a.e. on } T, \quad y(0)=y(b) .
$$

So $\left|y^{\prime}(\cdot)\right|^{p-2} y^{\prime}(\cdot) \in W^{1, r}(T)$ with $r=\min \left\{q, r^{\prime}\right\}$, hence $\left|y^{\prime}(\cdot)\right|^{p-2} y^{\prime}(\cdot) \in C(T)$, from which we have $y^{\prime} \in C(T)$. Therefore $y \in C^{1}(T)$. Also from (9), for every $u \in W_{p e r}^{1, p}(T)$ we have

$$
\begin{aligned}
\int_{0}^{b}\left|y^{\prime}(t)\right|^{p-2} y^{\prime}(t) u^{\prime}(t) d t=\int_{0}^{b} f(t, y(t)) u(t) d t \\
\Rightarrow\left|y^{\prime}(b)\right|^{p-2} y^{\prime}(b) u(b)-\left|y^{\prime}(0)\right|^{p-2} y^{\prime}(0) u(0) \\
\quad-\int_{0}^{b}\left(\left|y^{\prime}(t)\right|^{p-2} y^{\prime}(t)\right)^{\prime} u(t) d t=\int_{0}^{b} f(t, y(t)) u(t) d t \text { integration by parts } \\
\Rightarrow\left|y^{\prime}(0)\right|^{p-2} y^{\prime}(0) u(0)=\left|y^{\prime}(b)\right|^{p-2} y^{\prime}(b) u(b) \text { for all } u \in W_{\text {per }}^{1, p}(T) \quad \text { (see (10)) } \\
\Rightarrow\left|y^{\prime}(0)\right|^{p-2} y^{\prime}(0)=\left|y^{\prime}(b)\right|^{p-2} y^{\prime}(b) \Rightarrow y^{\prime}(0)=y^{\prime}(b) .
\end{aligned}
$$

Therefore $x_{1}, x_{2} \in C^{1}(T)$ are the two distinct nontrivial solutions of (1) with $\left|x_{i}^{\prime}(\cdot)\right|^{p-2} x_{i}^{\prime}(\cdot) \in W_{p e r}^{1, r}(T)$.

\section{ACKNOWLEDGEMENT}

The authors thank the referee for his corrections on the first version of the paper.

\section{REFERENCES}

[1] R. Adams: Sobolev Spaces, Academic Press, New York (1975). MR 56:9247

[2] P.Bartolo-V.Benci-D.Fortunato: Abstract critical point theorems and applications to some nonlinear problems with strong resonance at infinity, Nonlinear Anal. 7 (1983), 981-1012. MR 85c: 58028

[3] K.-C. Chang: Infinite Dimensional Morse Theory and Multiple Solution Problems, Birkhäuser, Boston (1993). MR 94e:58023 
[4] H.Dang-S.F.Oppenheimer: Existence and uniqueness results for some nonlinear boundary value problems, J. Math. Anal. Appl. 198 (1996), 35-48. MR 96m:34033

[5] M.Del Pino-R.Manasevich-A.Murua: Existence and multiplicity of solutions with prescribed period for a second order quasilinear ode, Nonlinear Anal. 18 (1992), 79-92. MR 92m:34055

[6] C.Fabry-D.Fayyad: Periodic solutions of second order differential equations with a $p$ Laplacian and asymmetric nonlinearities, Rend. Istit. Mat. Univ. Trieste 24 (1992), 207-227. MR 96b:34027

[7] Z. Guo: Boundary value problems of a class of quasilinear ordinary differential equations, Diff. Integral Eqns. 6 (1993), 705-719. MR 94d:34029

[8] S.Hu-N.S.Papageorgiou: Handbook of Multivalued Analysis. Volume I: Theory, Kluwer, Dordrecht, The Netherlands (1997). MR 98k:47001

[9] S.Kyritsi-N.Matzakos-N.S.Papageorgiou: Periodic problems for strongly nonlinear second order differential inclutions, J. Diff. Eqns. 183 (2002), 279-302.

[10] R.Manasevich-J.Mawhin: Periodic solutions for nonlinear systems with $p$-Laplacian like operators, J. Diff. Eqns. 145 (1998), 367-393. MR 99c:34034

[11] J. Mawhin: Periodic solutions of systems with p-Laplacian-like operators in Nonlinear Analysis and Applications to Differential Equations, Lisbon (1997), Progress in Nonlinear Differential Equations and Applications, Birkhäuser, Boston (1998). MR 2002d:34029]

[12] J.Mawhin-M.Willem: Critical Point Theory and Hamiltonian Systems, Springer-Verlag, New York (1989). MR 90e:58016

[13] M. Struwe: Variational Methods, Springer-Verlag, Berlin (1990). MR 92b:49002

Department of Mathematics, National Technical University, Zografou Campus, Athens 15780, Greece

Department of Mathematics, National Technical University, Zografou Campus, Athens 15780, Greece

E-mail address: npapg@math.ntua.gr 